\title{
Comparison of the efficacy and safety of oral fluconazole and topical clotrimazole in patients with candida balanitis
}

\author{
A Stary, J Soeltz-Szoets, C Ziegler, G R Kinghorn, R B Roy
}

One hundred fifty seven men with candidal balanitis were entered in a randomised, open-label parallel-group multicentre study comparing efficacy and safety of a single oral 150-mg fluconazole-dose with clotrimazole applied topically twice daily for 7 days. Of 64 fluconazole and 68 clotrimazole treated patients who were evaluable at short term follow up, $92 \%$ and $91 \%$ respectively were clinically cured or improved. Candida albicans was eradicated in $78 \%$ and $83 \%$ of patients respectively. Median time to relief of erythema was 6 days for fluconazole and 7 days for clotrimazole. Twelve of 15 patients who had received previous topical therapy for balanitis said they preferred oral therapy. At the one month follow up visit, 24/36 and 29/33 patients in the two groups were clinically cured or improved. Nine in the fluconazole group experienced a relapse; 6 of these 9 patients reported previous episodes of this infection during the past year. Two patients in the clotrimazole group had a relapse; neither had a history of previous episodes. Mycological eradication was noted in 26/36 and 25/33 patients in the two groups. Both treatment regimens were well tolerated. Thus a single $150 \mathrm{mg}$ dose of fluconazole was comparable in efficacy and safety to clotrimazole cream applied topically for 7 days when administered to patients with balanitis.

(Genitourin Med 1996;72:98-102)

Keywords: Fluconazole; clotrimazole; candida; candidiasis; balanitis

\section{Introduction}

Balanitis is an acute or chronic inflammation of the glans penis. The typical case is mild with erythema and symptoms of pain and burning. ${ }^{12}$ However, patients with balanitis can also present with profuse subpreputial discharge, oedema, and various degrees of phimosis. The microorganism most often cultured in symptomatic patients is Candida albicans. ${ }^{3-7}$ There are several factors that predispose patients to the development of balanitis including intercourse with an infected partner, recent antibiotic therapy, and poorly controlled diabetes mellitus. ${ }^{349}$ Additionally, balanitis commonly occurs in uncircumcised men. 5910

Conventional treatment of candida balanitis consists of topical application of an antifungal cream for a period of 1 to 2 weeks. ${ }^{45}$ Topical agents are messy and tend to rub off on clothing, which can lead to noncompliance. An alternative to topical therapy is short-course systemic therapy with an oral antifungal drug. Oral treatment tends to result in better patient compliance than topical creams. Better patient compliance to therapeutic regimens usually results in a better overall response to therapy. This is especially important in general practice where compliance tends to be less than in formal clinical studies. In this regard, fluconazole has been considered because of its favourable absorption profile, extensive distribution into tissues, and its lack of serious adverse effects. ${ }^{1112}$ Single-dose treatment with oral fluconazole is becoming well-accepted as a safe and effective alternative to topical treatment of candida vaginitis. ${ }^{13}$

To date, the only published study evaluat- ing single-dose fluconazole in patients with balanitis is a pilot open-label noncomparative study. ${ }^{14}$ The purpose of the present study was to compare the efficacy and safety of a single oral $150-\mathrm{mg}$ dose of fluconazole with that of multiple-dose topical clotrimazole $1 \%$ cream in patients with candida balanitis.

\section{Methods}

\section{Study Design}

This was a randomised, open-label, parallelgroup multicentre study. Male outpatients, 18 years of age or older, with a clinical and microbiological diagnosis of balanitis were eligible to enroll in the study. Signs and symptoms (mild, moderate, severe) of balanitis recorded included: white plaques, erythema, soreness, phimosis and fungal cultures. As culture results often require several days, there were some patients entered who clinically appeared to have balanitis, but cultures were negative (for unknown reasons). Those patients were thus not considered fully evaluable because a fungal pathogen was not isolated at baseline visit; however, clinical response (signs and symptoms) were recorded and thus reported. Excluded were patients with urogenital infection other than balanitis, a history of allergy to azole antifungals, impaired renal or hepatic function, and a history of alcoholism, drug abuse or psychological problems. Other reasons for exclusion from the study were participation in other investigational drug studies within the previous month, donation of blood or receipt of a blood transfusion within the previous 2 weeks, and treatment with any antifungal drug within 
the previous 2 weeks, which was assumed to be an adequate time period to eliminate the presence of any residual previous topical or oral antifungal therapy. Concomitant treatment with nonstudy antifungal drugs was prohibited during the trial period. A total of four investigational centres located in the United Kingdom (two) and in Austria (two) participated in the study. Study participants gave informed consent in accordance with the Declaration of Helsinki, and local ethical committee approval was obtained.

Eligible patients were randomly assigned to receive treatment with either a single oral 150 $\mathrm{mg}$ dose of fluconazole or clotrimazole $1 \%$ cream applied twice daily (morning and evening) for 7 days. At the baseline visit, before initiation of study drug therapy, all patients underwent a physical examination and a medical history was obtained. Previous episodes of balanitis, recent antifungal drug therapy, and whether or not the patient was circumcised were recorded. Pertinent information about the signs, symptoms, and treatment of any vaginal condition of the patient's partner was also recorded. Patients were evaluated for the clinical signs and symptoms of balanitis, including soreness, erythema, phimosis and white plaques. Each sign and symptom was graded as absent, mild, moderate, or severe. Other signs and symptoms not included in this list could also be recorded and graded. A preputial swab was obtained for microscopy and culture. Patients received a diary card on which to record the presence or absence of signs/symptoms for the first 7 days of the study. In addition, clotrimazole patients recorded the date and time of application of clotrimazole cream. The single dose of fluconazole was administered to fluconazole patients in the clinic. Patients were instructed as to proper hygiene and also to refrain from sexual intercourse until after the first followup visit.

Clinical and mycological evaluations were repeated at follow-up visits scheduled 8 to 11 days (short-term follow-up) and 28 to 32 days (long-term follow-up) after the baseline visit. The latter was selected as a practical time after this relatively minor infection that was thought to rely on patients to return. Beyond one month, it was thought that many more patients in this population would be lost to follow up. At each follow-up visit patients

Table 1 Demographic and infection characteristics of 157 patients with balanitis

\begin{tabular}{|c|c|c|}
\hline & $\begin{array}{l}\text { Fluconazole } \\
(n=81)\end{array}$ & $\begin{array}{l}\text { Clotrimazole } \\
(n=76)\end{array}$ \\
\hline \multicolumn{3}{|l|}{ Age (years) } \\
\hline Mean (range) & $33 \cdot 1(19-78)$ & $34.5(18-72)$ \\
\hline Patients with previous balanitis & $38(47 \%)^{\star}$ & $21(28 \%)$ \\
\hline \multirow{2}{*}{\multicolumn{3}{|c|}{ Duration of current episode (days) }} \\
\hline \multirow{2}{*}{\multicolumn{3}{|c|}{ Signs/symptoms }} \\
\hline & & \\
\hline Erythema & $72(89 \%)$ & $72(95 \%)$ \\
\hline Soreness & $53(65 \%)$ & $51(67 \%)$ \\
\hline White plaques & $45(56 \%)$ & $45(59 \%)$ \\
\hline Phimosis & $14(17 \%)$ & $17(22 \%)$ \\
\hline Mean severity score $f$ & 3.9 & $4 \cdot 1$ \\
\hline Patients with partners with vaginitis & $35(43 \%)$ & $33(43 \%)$ \\
\hline
\end{tabular}

were also evaluated for the occurrence of adverse events. During the short-term followup visit, diary cards were collected and patients were queried as to their abstinence from sexual intercourse since the baseline visit. Patients in the fluconazole group who had received previous topical antifungal therapy were asked to rate their preference for either oral or topical treatment.

\section{Microbiological technique}

Two dry cotton wool swabs were used to obtain samples for mycological analysis from different sites on the prepuce. The swab was plated onto Sabouraud's medium or Rice agar and incubated at $37^{\circ} \mathrm{C}$. Candida albicans was identified using a germ tube test. Other candida species were identified using the API 20C system (Analatab Products Inc, Plainview, NY).

\section{Efficacy evaluation}

Clinical efficacy was categorised as cure (disappearance of all baseline signs and symptoms of infection), improvement (partial disappearance of baseline signs and symptoms), or failure (no change or worsening). Improvement or cure followed by reappearance or worsening of clinical symptoms at the long-term follow-up was categorised as a relapse. Mycological efficacy was categorised as eradication (negative post-treatment culture result for candida) or persistence (a positive posttreatment candida culture). Eradication with reinfection was defined as eradication of candida at the short-term follow-up visit followed by reappearance of candida at the long-term follow-up visit.

\section{Statistical analysis}

Demographic characteristics and duration of infection were compared using the $t$ test. The distributions of clinical and mycologic response rates at short-term and long-term follow-up were compared between treatment groups using a chi-square test with continuity correction. They were also compared using as a baseline factor whether or not the patients had suffered a previous episode of balanitis using the Mantel-Haenszel test.

\section{Results}

A total of 157 patients were enrolled in the study and randomly assigned to one of the two treatment groups. Eighty one patients received fluconazole and 76 received clotrimazole. The two treatment groups were similar in terms of demographic characteristics (table 1), except that a significantly $(\mathrm{p}<0.05)$ higher percentage of patients in the fluconazole group (47\%) had experienced a previous episode of balanitis as compared with those in the clotrimazole group $(28 \%)$. This difference was taken into consideration when response rates were compared between treatments; however, the numbers were too small to establish a significant relationship with outcome. In the fluconazole group one patient was HIV positive, one had type II diabetes mellitus, and one had viral 
Table 2 Patient status at short-term and long-term evaluations and reasons for exclusion from efficacy analysis

\begin{tabular}{lrl}
\hline & Number of patients & \\
\cline { 2 - 3 } & Fluconazole $(n=81)$ & Clotrimazole $(n=76)$ \\
\hline Short-term clinical evaluation & & \\
(8-11 days) & 12 & 4 \\
Lost to follow-up & 1 & 1 \\
Withdrawn from study & 1 & 1 \\
No data at this visit & 3 & 2 \\
No baseline signs/symptoms of infection & 64 & 68 \\
Total evaluable & & \\
Short-term mycological evaluation & 12 & 4 \\
(8-11 days) & 1 & 2 \\
Lost to follow-up & 1 & 5 \\
Withdrawn from study & 4 & 64 \\
No data at this visit & 63 & \\
Negative baseline mycology & & 33 \\
Total evaluable & & 33 \\
Total evaluable at long-term evaluation & 36 & \\
(28-32 days) & 36 & \\
Clinical & Mycological &
\end{tabular}

^In addition, seven fluconazole patients and two clotrimazole patients who had no follow-up dat also had negative baseline mycology results; these patients are not counted again in this category.

warts. Two patients in the clotrimazole group had type II diabetes mellitus. None of the patients were circumcised before study entry; one patient underwent circumcision during the study period. Forty three per cent of the patients in both treatment groups reported having a partner with vaginitis.

The most common sign or symptom reported at baseline was erythema, reported by 72 patients in each treatment group (table 1). Others included, in descending order of frequency, soreness, white plaques, and phimosis. There was no difference between the groups in terms of the severity of clinical signs and symptoms at baseline. Most were reported as mild or moderate.

At the short-term follow-up visit, $64 \mathrm{flu}$ conazole-treated patients and 68 clotrimazoletreated patients had evaluable efficacy data. Reasons for exclusion from the efficacy analysis were as follows: (1) patient lost to follow-up after the baseline visit; (2) patient withdrew from study; and (3) no baseline signs or symptoms were recorded. These are summarised in table 2 along with the numbers of patients excluded in each treatment group. Regarding the higher rate of loss to follow-up in the fluconazole group (12 patients) than in the clotrimazole group (4 patients) it can only be hypothesised that patients who did not return

Figure 1 Clinical therapy with either singledose oral fluconazole 150 mg or topical clotrimazole twice daily for 7 days at short-term follow-up ( 8 to 11 days after baseline). ( $p$ = not significant)

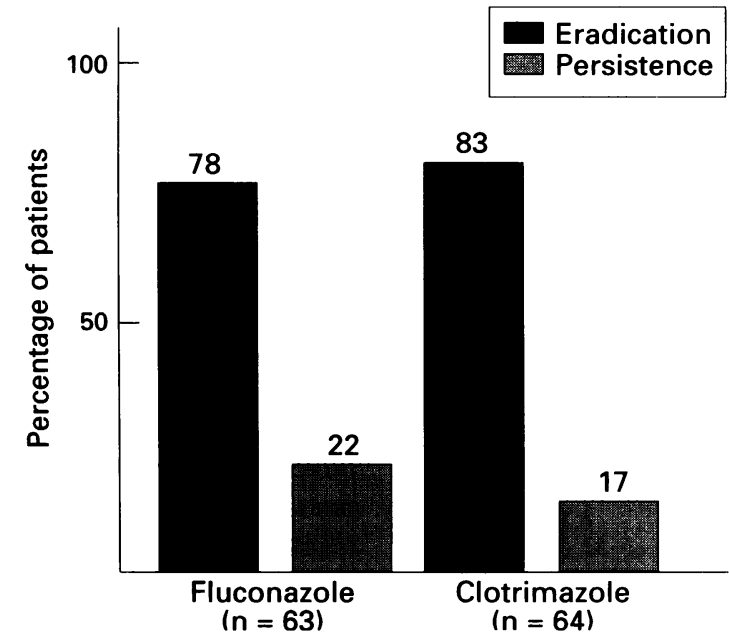

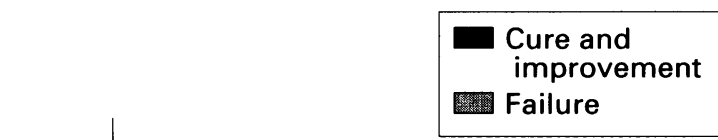
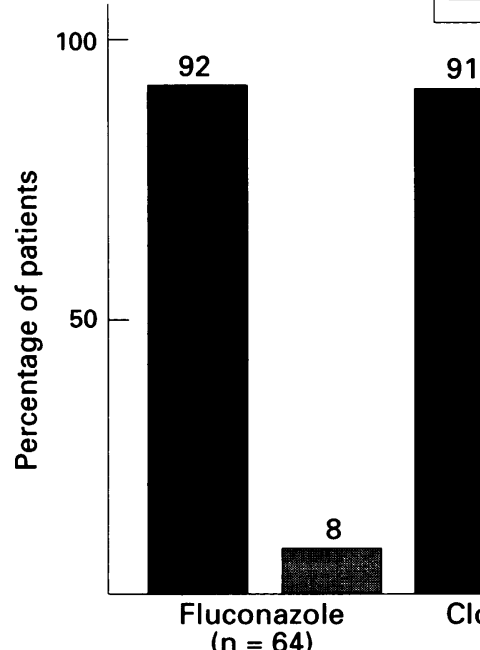

Figure 2 Mycologic response to antifungal therapy with either single-dose oral fluconazole $150 \mathrm{mg}$ or topical clotrimazole twice daily for 7 days at short-term follow-up ( 8 to 11 days after baseline). ( $p=$ not significant).

for further medical assessment were likely tơ be satisfied with their therapeutic outcome. If patient was dissatisfied with response to ther: apy, he would be more likely to seek additional therapy. Of the two patients who were withdrawn from the study, one had a bacterial infection (fluconazole group) and one undero went a pancreatectomy (clotrimazole group) Fifty nine of the $64(92 \%)$ fluconazole-treated patients and 62 of the $68(91 \%)$ clotrimazole treated patients were clinically cured or improved at short-term follow-up (fig 1). Thiş difference was not statistically significant.

Of the 157 patients enrolled in the study? 139 (70 fluconazole; 69 clotrimazole) had a positive culture result for $C$ albicans. In addi tion, two patients also had other organismș present at baseline: Torulopsis glabrata (one fluconazole patient) and Torulopsis sp (one clotrimazole patient). For analysis of the short-term mycological data, four patients its the fluconazole group and five in the clotrima? zole were excluded because of a negative base line culture result for $C$ albicans (table 2) Thus 63 fluconazole patients and 64 clotriman zole patients had evaluable mycology data as short-term follow-up. In $49(78 \%)$ patients treated with fluconazole and $53(83 \%)$ treateç with clotrimazole, the $C$ albicans was eradicated (fig 2). This difference was not statisti꾹 cally significant. The fluconazole-treated patient with $T$ glabrata showed clinicas improvement but due to persistence of this organism was subsequently treated with clotri mazole/hydrocortisone. The Torulopsis $s p$ was eradicated.

Based on the patient's self assessment, the median time to relief of erythema for the $12 \overline{6}$ patients ( 59 fluconazole; 67 clotrimazole) who reported it and returned for follow-up was 6 days in the fluconazole group and 7 days in the clotrimazole group. This difference was not statistically significant. Of the 15 fluconazole-treated patients who had previously 
received topical therapy for balanitis, 12 $(80 \%)$ said they preferred the oral therapy.

At the long-term follow-up visit, several patients did not return for evaluation, some did not return as scheduled, and others received nonstudy antifungal therapy after the short-term visit even though they were clinically cured or improved at this visit. Thus, 36 fluconazole-treated patients and 33 clotrimazole-treated patients were evaluable for efficacy (table 2). Twenty four (67\%) patients treated with fluconazole and $29(88 \%)$ treated with clotrimazole were clinically cured or improved. There were nine relapses and three failures in the fluconazole group; two relapses and two failures occurred in the clotrimazole group. Seven of the nine patients in the fluconazole group who reported clinical relapse had mycologic confirmation of infection; two patients had a negative mycologic culture result. Both patients in the clotrimazole group who reported clinical relapse had mycologic confirmation of infection. Of the 36 evaluable fluconazole patients and 33 evaluable clotrimazole patients, $26(72 \%)$ and $25(76 \%)$ patients, respectively, had a mycologic response of eradication. Reinfections in six patients and persistence in four patients were reported in the fluconazole group. A total of eight clotrimazole-treated patients had reinfection or persistence (three and five patients, respectively). Six (four fluconazole; two clotrimazole) of the nine patients with reinfection admitted to having sexual intercourse between visits.

Both treatment regimens were well tolerated. Only one patient in the fluconazole group reported mild diarrhoea 2 days after ingesting the single dose. The diarrhoea resolved spontaneously.

\section{Discussion}

Vulvovaginal candidiasis is a common infection in women; more recently, the importance of genital candidiasis in men has become recognized..$^{815}$ Over the 10 year period from 1976 to 1986 , the number of men with genital candidiasis presenting to a genitourinary clinic in the United Kingdom increased by $75 \% .{ }^{14}$ This infection is frequently associated with a concurrent vaginal candidiasis infection in a sexual partner. The moist epithelium below the prepuce provides ideal growing conditions for microorganisms, especially in uncircumcised men. Bacteria and yeast, especially candida, are abundant in the preputial sac. Although candida is usually saprophytic, this organism may become pathogenic under conditions of lowered local or general resistance. ${ }^{18}$

Although candidal balanitis is not a serious infection, the distressing symptoms and associated discomfort necessitate rapid and effective treatment. As is evident from our study, as well as previously published balanitis treatment studies, ${ }^{15-18}$ the patient's motivation for follow-up evaluation and treatment disappears rapidly. Once the patient realises he has a relatively benign condition and the anxiety of having an abnormal genital condition resolves, compliance with follow-up visit schedules decreases. Therefore, a therapy that provides high cure rates with minimal adverse effects after a single dose is ideal for these patients.

A number of topical and systemic antifungal drugs are available for the treatment of candida balanitis. Topical application with the polyene antifungals, nystatin ${ }^{16}$ and natamycin, ${ }^{17}$ and the imidazole and triazole antifungals, bifonazole, ${ }^{18}{ }^{19}$ butaconazole, ${ }^{20}$ clotrimazole, ${ }^{1518}$ and miconazole, ${ }^{16}$ have produced excellent results with short-term mycologic response rates in the range of $77 \%$ to $100 \%$. All of the topical therapies, however, require 1 to 2 weeks of application for effective cures, and patient compliance is hampered by the necessary prolonged course of therapy.

The newer azole antifungal agents have been developed to provide less frequent application and shorter courses of treatment while maintaining the efficacy rates observed with the older drugs. This goal has been achieved with the use of single-dose oral fluconazole in the treatment of vulvovaginal candidiasis. ${ }^{13}$ Fluconazole has several characteristics that are appealing in the treatment of genital candidiasis. It is well-absorbed following oral administration, it has a long elimination half-life, and it is primarily excreted unchanged in the urine. ${ }^{11} 1221$ Because of its low lipophilicity and low degree of protein binding, fluconazole penetrates body tissues and fluid rapidly and efficiently. For example, high concentrations of fluconazole are achieved in vaginal tissue. ${ }^{11}$ In addition, fluconazole is well tolerated, with a good safety profile. ${ }^{21}$

In their preliminary investigation of 14 men who presented with candida balanitis, Kinghorn and Woolley ${ }^{14}$ reported a $100 \%$ mycologic cure rate in 11 men who returned for follow-up evaluation after a single $150 \mathrm{mg}$ dose of fluconazole. All 11 patients experienced symptomatic improvement without adverse effects. In our randomised multicentre controlled study, we found no significant difference in cure rates between topical clotrimazole $1 \%$ cream applied twice daily for 7 days and a single $150 \mathrm{mg}$ dose of fluconazole. Of the 15 patients who had previously received topical antifungal treatment, 12 said they preferred oral fluconazole.

Although the clinical response rate at longterm follow-up was higher for clotrimazole than for fluconazole, there was little difference between treatment groups in the mycologic eradication rate. It is unclear why more clinical relapses occurred 4 weeks after therapy in patients treated with fluconazole, a systemically active agent, as compared with those treated with topical clotrimazole. Several factors, however, should be considered when evaluating the difference in outcomes between the two treatment groups. Our first consideration was that the number of patients with previous episodes of candida balanitis was significantly higher in the fluconazole group. Six of the nine $(67 \%)$ patients who experienced clinical relapse in the fluconazole group had previous episodes of balanitis. All of these 
patients had at least two episodes during the previous 12 months. None of the clotrimazole patients in whom clinical relapse occurred reported episodes of balanitis during the previous 12 months. Secondly, of the 10 patients with relapse/reinfection at long-term followup, four fluconazole-treated but only two clotrimazole-treated patients reported having had sexual intercourse during the time between the last two visits. The importance of treating both partners simultaneously is generally recognised. ${ }^{15}$ Thirdly, it is possible that patients in the clotrimazole group practiced better hygiene because they were applying topical medication twice a day. Perhaps future investigations should place greater emphasis on good hygiene, particularly for the benefit of those receiving oral therapy. Another consideration was that perhaps mycologic responders and nonresponders should be compared with respect to fluconazole dose on the basis of mg-per-kg body weight or body mass. Indeed, at the short-term visit heavier patients were more likely to have a response of persistence than eradication. The mean weight of fluconazole-treated patients in whom candida was eradicated was $77.5 \mathrm{~kg}$ compared with $83.8 \mathrm{~kg}$ for patients who had a response of persistence $(p=0.02)$. A final consideration is that global ratings may be inappropriate for detecting small differences in efficacy.

In summary, we found similar clinical and mycologic response rates to treatment with either a single oral $150-\mathrm{mg}$ dose of fluconazole or topical clotrimazole $1 \%$ cream administered twice daily for 7 days to patients with candida balanitis. Clinical cure or improvement occurred in over $90 \%$ of evaluable patients treated with either fluconazole or clotrimazole, and mycologic eradication rates were comparably high. Both treatments were well-tolerated. Fluconazole given as a single oral dose is a more convenient therapy than conventional multiple-dose topical regimens. These factors may contribute to improved patient acceptability and compliance. Future studies may further define the role of singledose fluconazole in the simultaneous treat- ment of sexual partners with candidal balanitis and vaginitis, and in the prevention of socalled "ping-pong" infections.

The study was funded by a grant from the participating institutions from Pfizer, the manufacturer of fluconazole. Data analysis was performed by the Pfizer clinical research unit.

1 Vohra S, Badlani G. Balanitis and balanoposthitis. Urol Clin North Am 1992;19:143-7.

2 Pariser DM. Cutaneous candidiasis. A practical guide fo primary care physicians. Postgrad Med 1990;87:101-8.

3 Abdullah AN, Drake SM, Wade AAH, Walzman $M$ Balanitis (balanoposthitis) in patients attending a department of genitourinary medicine. Int $\mathcal{F} S T D$ AIDS 1992; 3:128-9.

4 Csonka GW. Balanitis. Clin Med 1974;81:15-9.

5 McCrossin ID. Genital skin disease: a guide to management in general practice. Mod Med Aust 1993;36: ment

6 Lefevre J-C, Lepargneur J-P, Bauriaud R, Bertrand M-A Blanc C. Clinical and microbiologic features of urethritis in men in Toulouse, France. Sex Transm Dis 1991;18: $76-9$

7 Forster GE, Harris JRW. A clinical and microbiological study of balanitis. Eur $\mathcal{F}$ Sex Transm Dis 1985;3:31-4

8 Wise GJ, Silver DA. Fungal infections of the genitourinar system. F Urol 1993;149:1377-88.

9 Waugh MA. Clinical presentation of candidal balanitis-its differential diagnosis and treatment. Chemotherapy 1982; 28(suppl 1):56-60.

10 Fakiian N, Hunter S, Cole GW, Miller J. An argument for circumcision. Prevention of balanitis in the adult. Arch Dercumatol 1990;126:1046-7.

11 Lazar JD, Hilligoss DM. The clinical pharmacology of fluconazole. Semin Oncol 1990;17(suppl 6):14-8.

12 Brammer KW, Farrow PR, Faulkner JK. Pharmacokinetic and tissue penetration of fluconazole in humans. Rev Infect Dis 1990;12(suppl 3):S318-26.

13 Patel HS, Peters MD, Smith CL. Is there a role for fluconazole in the treatment of vulvovaginal candidiasis? Ann Pharmacother 1992;26:350-3.

14 Kinghorn GR, Woolley PD. Single-dose fluconazole in the treatment of Candida albicans balanoposthitis. Int f STD AIDS 1990;1:366-7.

15 Waugh MA, Evans EGV, Nayyar KC, Fong $R$ Clotrimazole (Canesten) in the treatment of candidal balanitis in men. With incidental observations on diabetic candidal balanoposthitis. Br $f$ Venereal Dis 1978;54: candidal

16 Forster GE, Harris JRW. Double blind therapeutic trial in balanitis-miconazole and nystatin. Eur $\mathcal{F}$ Sex Trans Dis 1986;3:81-3

17 Masterton G, Sengupta SM, Schofield CBS. Natamycin in genital candidosis in men. Br $\mathcal{F}$ Venereal Dis 1975;51 $210-2$

18 Maw RD, Horner T, Evans J. A comparative trial of bifonazole $1 \%$ cream and clotrimazole $1 \%$ cream in the treatment of candidal balanoposthitis. Mykosen 1987;30 229-32.

19 Tosi E, Bertani E, Pavone PS, Bianchi W, Viscovo R. 1\% bifonazole cream in the treatment of candida balanitis: a clinical trial. Eur Rev Med Pharmacol Sci 1987;9:265-7.

20 Nolting S. An open study of butoconazole nitrate $2 \%$ cream in patients with candidal balanoposthitis or balanicream in patients with candidal balan

21 Como JA, Dismukes WE. Oral azole drugs as systemic antifungal therapy. $N$ Engl f Med 1994;330:263-72. 